\title{
RELAÇÃO ENTRE A QUALIDADE DE SEMENTES DE AVEIA-PRETA E A PRODUÇÃO DE FORRAGEM E DE SEMENTES
}

\section{RELATION BETWEEN SEED PHYSIOLOGICAL QUALITY OF BLACK OAT AND FORAGE AND SEEDS PRODUCTION}

\author{
Luis Osmar BragaSCHUCH ${ }^{1}$ \\ Eliane Maria KOLCHINSKI ${ }^{2}$ \\ Leandro Damero CANTARELLI ${ }^{3}$
}

\begin{abstract}
RESUMO
Com objetivo de avaliar o efeito da qualidade fisiológica das sementes de aveia preta (Avena strigosa Schreb) sobre a produção de forragem, a capacidade de rebrote da planta e o rendimento de sementes foi conduzido um experimento no ano de 2000, em Pelotas, RS. Foram avaliados cinco níveis de qualidade fisiológica das sementes e dois sistemas de manejo das plantas (com corte e sem corte) utilizando o cultivar Preta Comum. O delineamento experimental utilizado foi de blocos ao acaso, com três repetições. O aumento da qualidade fisiológica de sementes proporciona aumentos no número de colmos, produção de matéria seca, índice de área foliar e rendimento de sementes. As diferenças na qualidade fisiológica de sementes não causam efeito sobre a capacidade de rebrota.
\end{abstract}

Palavras-chave: Avena strigosa; produção de sementes; matéria seca; área foliar.

\begin{abstract}
With the objective to evaluate the effect of seed physiological quality on the forage production, regrowth after the cutting and seeds yield of black oats (Avena strigosa Schreb), a field experiment was conducted in the year, in Pelotas, Rio Grande do Sul State, Brazil. This study evaluated five levels of seed physiological quality and two systems management (without cutting/with cutting) of the plants, using black oats common cultivars. Were used the experimental design was a randomized block with three replication. Increases in levels of seed physiological quality provided increments the stems $\mathrm{m}^{2}$, the dry matter production, leaf area index and seed yields. The variation the seed physiological quality did not affect the regrowth capacity of the plants.
\end{abstract}

Key-words: Avena strigosa; seeds production; dry matter; leaf area.

\footnotetext{
1 Engenheiro Agrônomo, Dr. em Agronomia, Professor Associado da Faculdade de Agronomia Eliseu Maciel, UFPel, Bolsista do CNPq. Caixa Postal: 345, CEP: 96001-970, Pelotas - RS. E-mail: lobs@ufpel.edu.br. Autor para correspondência.

${ }^{2}$ Eng. Agrônomo, Doutora em Agronomia, Professora adjunta da Universidade Estadual do Rio Grande do Sul. Rua Alegrete, 821. Bairro São José. CEP: 95960-000, Encantado-RS. E-mail: eliane-kolchinski@uergs.edu.br.

${ }^{3}$ Engenheiro Agrônomo, Mestre em Ciência e Tecnologia de Sementes. Responsável Técnico na Cevale. Rua Praça Paris, no 142, Bairro Jardim Europa, CEP: 85935-000, Assis Chateaubriand - PR. E-mail: cantarellil@bol.com.br.
} 


\section{INTRODUÇÃO}

Nos estados do sul do Brasil, a aveia é a principal forrageira utilizada na formação de pastagens de inverno. Isso ocorre devido à sua alta produção de matéria seca, qualidade nutritiva da forragem, resistência ao pisoteio e ao baixo custo de produção (FLOSS et al., 1999). Estima-se que no Estado do Rio Grande do Sul são cultivados aproximadamente 1.250 .000 ha de aveia com fins forrageiros (FLOSS, 2001), utilizados na alimentação de gado leiteiro, terminação de bovinos de corte e na produção de ovinos. Esse procedimento auxilia no suprimento da alimentação dos rebanhos, numa época em que ocorre insuficiência de pastos.

$\mathrm{Na}$ implantação de pastagens é imprescindível o uso de sementes de alta qualidade fisiológica. Entretanto, a produção de sementes tem sido realizada muitas vezes, por produtores não especializados, que não observam determinadas práticas agronômicas importantes para a obtenção de sementes de alta qualidade. Nesse contexto, temse constatado que as sementes de aveia-preta utilizadas no Rio Grande do Sul apresentam baixa qualidade. Estima-se que a maioria das sementes utilizadas para semeadura no Estado tem 60 e $70 \%$ de germinação (SCHUCH, 1999).

A qualidade fisiológica das sementes, representada pela germinação e pelo vigor, pode afetar a capacidade das sementes em regenerar a planta. Efeitos da qualidade fisiológica das sementes sobre a velocidade e uniformidade de emergência, emergência total e estabelecimentos das plantas têm sido documentados (SCHUCH e LIN, 1982a; SCHUCH e LIN, 1982b). MACHADO (2002) constatou que a redução progressiva da qualidade fisiológica das sementes provocou reduções e causou desuniformidade da emergência das plântulas em campo. SCHUCH (1999) observou que o uso de sementes com maior qualidade fisiológica produziram plantas com maior tamanho inicial, o que conseqüentemente proporcionou maiores taxas de crescimento da planta, de produção de matéria seca e da área foliar ao longo do período inicial de crescimento.

$\mathrm{Na}$ maioria dos trabalhos, os efeitos significativos da qualidade fisiológica das sementes sobre o rendimento de sementes em cultivos anuais, normalmente estão associadas à inadequação da densidade da população (SCHUCH e LIN, 1982b). Segundo BURRIS (1976) e ROBERTS (1986), a relação entre qualidade fisiológica das sementes e o rendimento depende se o rendimento foi avaliado no estágio vegetativo ou reprodutivo. Plantas colhidas durante o crescimento vegetativo ou reprodutivo precoce têm usualmente mostrado relacionamento positivo entre o vigor de sementes e o rendimento, o mesmo não ocorre para as plantas colhidas na maturação. Nesse sentido, TEKRONY et al. (1989) observaram que plantas de milho provenientes de sementes de baixa qualidade fisiológica apresentaram redução do crescimento vegetativo, especialmente no estágio do colar da quarta folha, mas não observados na época do embonecamento. Em condições com população adequada de plantas, não houve relação entre qualidade fisiológica de sementes e rendimento.

O cultivo da aveia com objetivo de produzir forragem está relacionado à colheita no estágio vegetativo ou reprodutivo precoce, uma vez que a planta pode ser submetida ao pastejo a partir do perfilhamento pleno (DENARDIN e KOCHLANN, 1983), e o momento para fenação ocorrerá quando apresentar altura em torno de $30 \mathrm{~cm}$, aos 60 dias após a emergência das plantas (SANTOS et al., 1990), situações em que são esperados mais efeitos da qualidade fisiológica das sementes. Desta forma, é provável que o uso de sementes de alta qualidade fisiológica na implantação de pastagens com aveia afete favoravelmente o desenvolvimento inicial das plantas, com efeitos positivos na produção de forragem.

Com base nessas considerações, 0 presente trabalho objetivou avaliar o efeito da qualidade fisiológica das sementes sobre 0 desempenho forrageiro e a produção de sementes de aveia-preta.

\section{METODOLOGIA}

O experimento foi conduzido em condições de campo no Centro Agropecuário da Palma da Universidade Federal de Pelotas - CAP/UFPEL, localizado no município de Capão do Leão - RS, em solo classificado como Argissolo Amarelo Distrófico típico (Tipic Paleudalf).

Os tratamentos constaram da combinação de cinco níveis de qualidade fisiológica de sementes e dois sistemas de manejo das plantas (com corte e sem corte) utilizando o cultivar Preta Comum. Os níveis de qualidade fisiológica das sementes distribuíram-se ao longo de um gradiente desde um nível muito alto, representado por um lote com $96 \%$, até um nível muito baixo, representado por um lote com $30 \%$ de germinação, estando entre esses dois extremos três níveis intermediários representados por lotes com germinações de $88 \%, 72 \%$ e $52 \%$.

Os diferentes níveis de qualidade fisiológica das sementes foram obtidos pela técnica de hidrotermoterapia modificada (BRATTACHARYYA et al., 1985), que consistiu na imersão das sementes de um lote de alta qualidade fisiológica em água aquecida a $50{ }^{\circ} \mathrm{C}$, por tempos variáveis: zero, 110 , 190, 245 e 310 min. Após o tratamento hidrotérmico as sementes foram submetidas ao teste de germinação (BRASIL, 1992) obtendo resultados de $96,88,72,52$ e $30 \%$.

A semeadura foi realizada sob sistema de plantio direto, em parcelas constituídas de nove linhas de cinco metros de comprimento espaçadas de $17 \mathrm{~cm}$, na densidade de 300 sementes de cada tratamento por metro quadrado. O delineamento experimental utilizado foi de blocos ao acaso com três repetições.

Aos 60 dias após a emergência da plântula (DAE), foi realizado um corte em metade de cada 
parcela, restando aproximadamente $7 \mathrm{~cm}$ da altura da planta. Nas plantas coletadas foram determinados o índice de área foliar (IAF) utilizando um determinador fotoelétrico e a produção de matéria seca pelo método estufa a $55^{\circ} \mathrm{C}$ por $72 \mathrm{~h}$. Antes do corte das plantas foi computado o número de colmos presentes em um metro de fileira, para a determinação do número de colmos metro quadrado.

Por ocasião da maturação, as determinações experimentais foram efetuadas nas áreas úteis em cada metade das parcelas, visando verificar o efeito da qualidade de sementes sobre as plantas após terem sido submetidas ao corte. Foram determinados o número de colmos por metro quadrado, a produção de matéria seca e o número de panículas por metro quadrado através da avaliação das plantas presentes em um metro de fileira; a estatura de plantas por meio da medição de 10 plantas ao acaso e o número de sementes por panícula utilizando 30 panículas em cada metade de parcela. O rendimento de sementes foi determinado pela colheita das áreas úteis, transformado em $\mathrm{kg} \mathrm{ha}^{-1}$ e corrigido para $13 \%$ de água.

Os dados experimentais foram submetidos à análise de variância. $O$ efeito do corte foi avaliado por comparações de médias, através do teste de Duncan ao nível de $5 \%$ de significância, enquanto o efeito do vigor por regressões polinomiais.

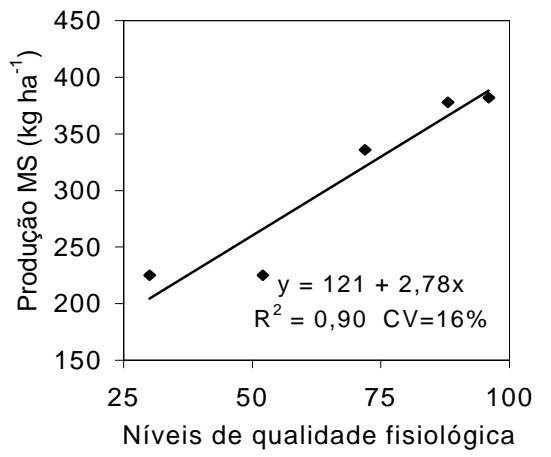

\section{RESULTADOS E DISCUSSÃO}

A avaliação das plantas aos 60 dias após a emergência (DAE) mostrou que o aumento no nível de qualidade fisiológica das sementes proporcionou acréscimos na produção de matéria seca e no IAF (Figura 1). Comparando o lote que apresentava qualidade fisiológica próxima ao limite mínimo aceito para comercialização de sementes de aveiapreta (lote com germinação de $72 \%$ ), com o lote de qualidade mais elevada, observou-se a elevação do IAF de 0,94 para 1,39, e da produção de matéria seca de $336 \mathrm{~kg} \mathrm{ha}^{-1}$ para $382 \mathrm{~kg} \mathrm{ha}^{-1}$, representando acréscimos de 48 e $13 \%$, respectivamente. Fazendo essa mesma comparação com o lote de qualidade fisiológica mais baixa os acréscimos são mais acentuados, com valores de $98 \%$ e $70 \%$ no IAF e na produção de matéria seca, respectivamente. Efeitos da qualidade fisiológica das sementes sobre a produção de matéria seca e IAF, também foram constatados em outros trabalhos (TEKRONY et al., 1989, SCHUCH et al., 2000, HOFS, 2003). Segundo DAN et al. (1987), sementes vigorosas apresentam maior capacidade de transformação e de suprimento de reservas dos tecidos de armazenamento e da maior incorporação desses pelo eixo embrionário. Isso pode resultar em emergência mais rápida e uniforme e plântulas maiores, proporcionando taxas superiores de crescimento no período inicial de desenvolvimento das plantas (SCHUCH, 1999; MACHADO, 2002; HÖFS, 2003), influenciando o IAF e o acúmulo de matéria seca.

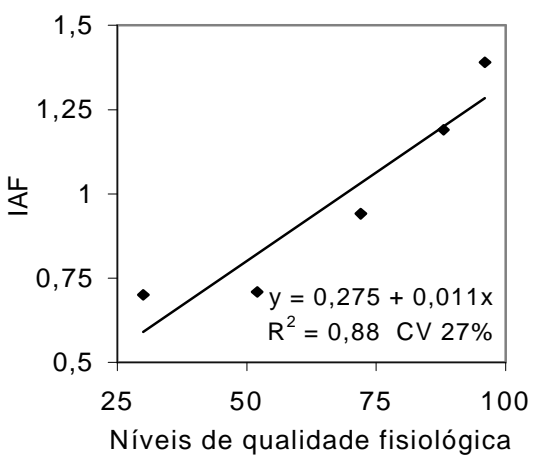

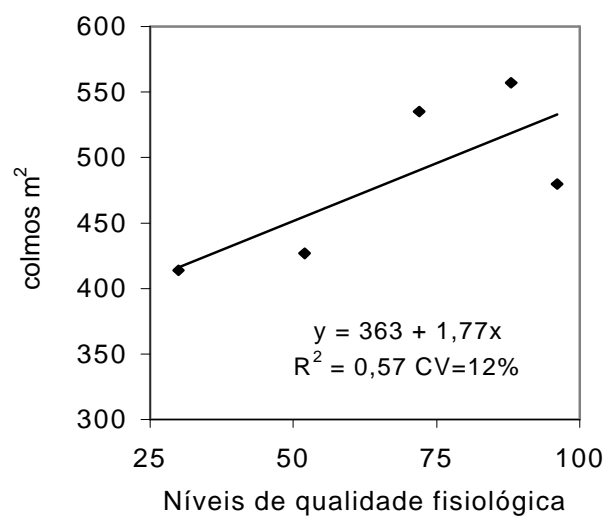

FIGURA 1 - Produção de matéria seca (MS), índice de área foliar (IAF) e número de colmos por $\mathrm{m}^{2}$ de plantas de aveia-preta aos 60 dias após a emergência em função de diferentes níveis de qualidade fisiológica de sementes. 
SCHUCH, L.O.B. et al. Relação entre a qualidade de sementes...

Os resultados observados na produção de matéria seca e no IAF demonstraram o efeito positivo da qualidade fisiológica de sementes sobre a produção inicial de forragem e a importância da utilização de sementes com alta qualidade na formação de pastagens. De acordo TEKRONY e EGLI (1991), plantas colhidas durante o crescimento vegetativo têm usualmente mostrado relacionamento positivo entre qualidade fisiológica de sementes e rendimento. Essas informações são importantes especialmente porque as sementes utilizadas na semeadura no Rio Grande do Sul apresentam, em geral, níveis de qualidade fisiológica relativamente baixos, com germinação entre $60-70 \%$ para a maioria dos lotes $(\mathrm{SCHUCH}$, 1999).

O uso de sementes de qualidade fisiológica baixa reduziu o número de colmos por metro quadrado (Figura 1), similar ao observado na maturação (Figura 2). Isso mostra que mesmo ajustando a densidade de semeadura, não foi possível obter o mesmo número de colmos em populações provenientes de sementes com níveis de qualidade diferentes. $O$ uso de populações mais elevadas de plantas pode compensar o efeito da baixa qualidade de sementes e a menor produção de matéria seca e IAF (Figura 1), conforme relatado por SCHUCH et al. (2000).

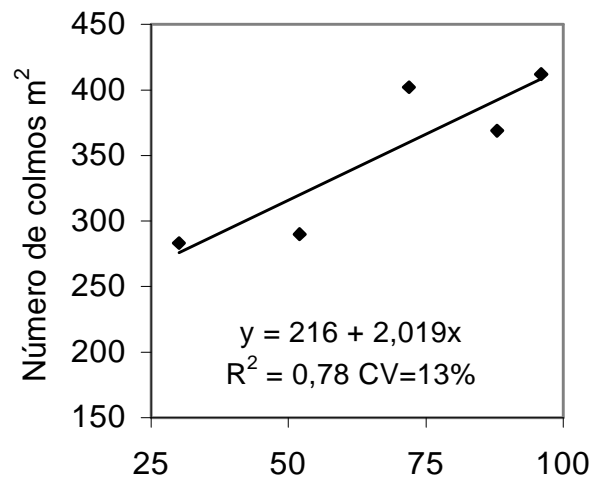

Níveis de qualidade fisiológica

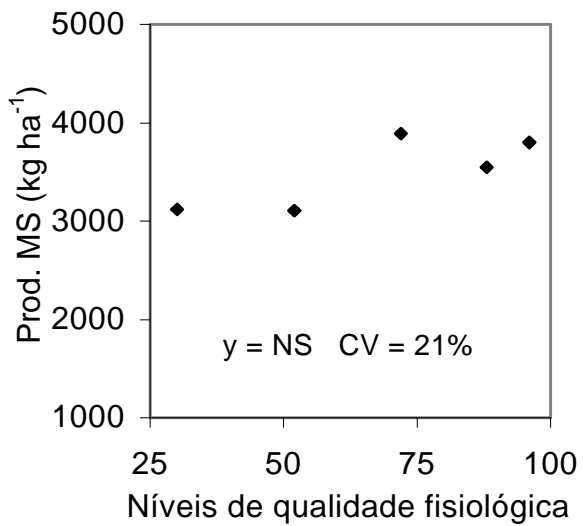

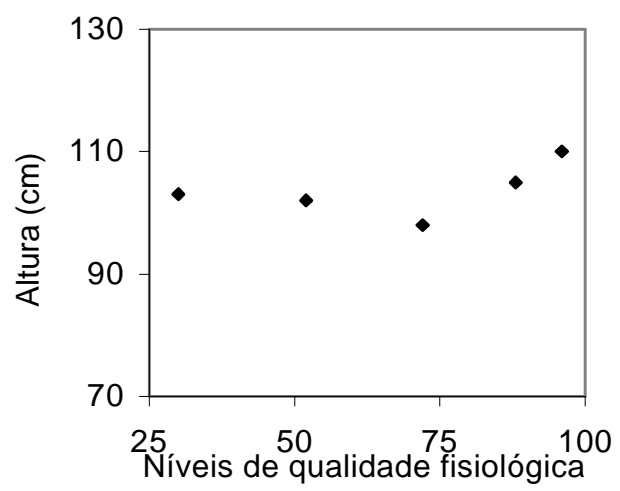

FIGURA 2 - Número de colmos por $\mathrm{m}^{2}$, produção de matéria seca (MS) e altura de plantas de aveia-preta na maturação (média com e sem corte) em função de diferentes níveis de qualidade fisiológica de sementes.

Não houve efeito da qualidade fisiológica de sementes sobre as plantas na maturação em função do sistema de manejo (com ou sem corte), exceto para o número de sementes por panícula e peso de mil sementes.

O efeito da qualidade fisiológica observado na produção de matéria seca aos 60 DAE não se manifestou na maturação (Figura 2). De acordo com a literatura, o efeito da qualidade fisiológica de sementes, observado no crescimento inicial das plantas tende a reduzir com a evolução do crescimento da mesma. SCHUCH et al. (2000) constaram que as diferenças de vigor das sementes afetaram a produção de matéria seca das plantas de aveia-preta até aos 75 DAE não verificado em avaliações posteriores. Segundo KHAN et al. (1989) em condições agrícolas normais, pode ser esperado que as vantagens iniciais da emergência precoce e do maior crescimento inicial da planta diminuam com o avanço no período de crescimento, em função da competição entre as plantas pelos fatores de produção (água, luz e nutrientes) iniciar mais cedo, em cultivos derivados de sementes de melhor qualidade. Esses resultados indicam a 
ausência de efeito da qualidade fisiológica das sementes sobre a capacidade de rebrote das plantas.

De forma semelhante à produção de matéria seca na maturação, a altura de plantas também não foi afetada pelos diferentes níveis de qualidade das sementes. Resultados semelhantes foram obtidos por SCHUCH et al. (1999) e MACHADO (2002).
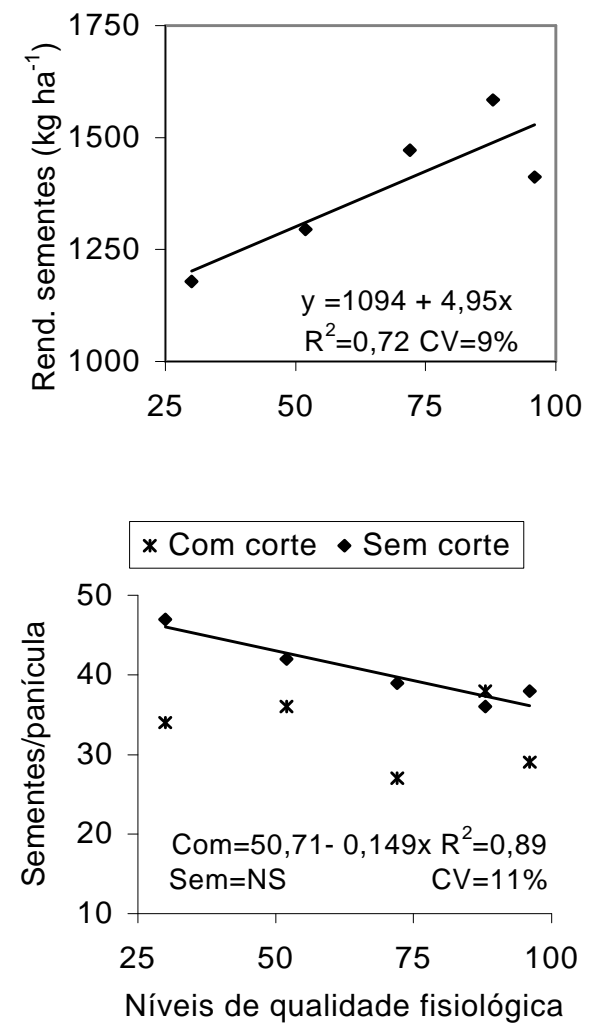

O uso de sementes com qualidade fisiológica superior incrementou o rendimento de sementes (Figura 3). Efeitos do vigor de sementes sobre o rendimento também foram constatados em outros trabalhos (MACHADO 2002; HÖFS, 2003). De acordo com KHAN et al. (1989), quando sementes com qualidade inferior são utilizadas (germinação inferior a 85\%) ocorre redução considerável no rendimento, até mesmo quando é realizado o ajuste na taxa de semeadura.

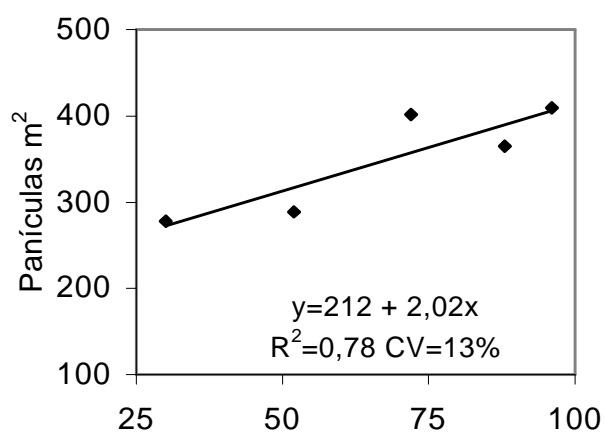

* Com corte $\bullet$ Sem corte

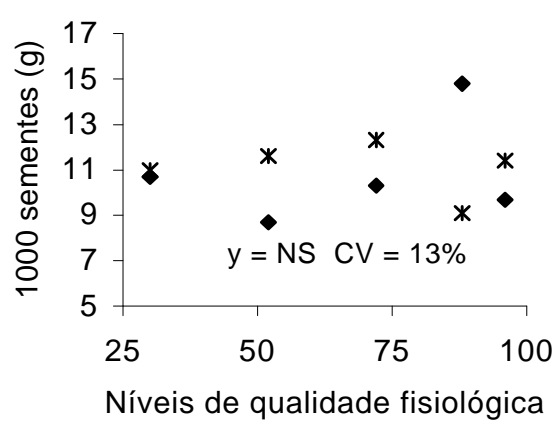

FIGURA 3 - Rendimento de sementes e componentes de rendimento de plantas de aveia-preta em função de diferentes níveis de qualidade fisiológica de sementes.

$O$ rendimento de sementes foi determinado pelo aumento no número de panícula por metro quadrado. O uso de sementes com maior qualidade fisiológica afetou negativamente o número de sementes por panículas quando não foi efetuado o corte e não exerceu efeito quando se efetuou o corte. Já o peso de mil sementes não diferiu entre os níveis de qualidade nos dois sistemas, com e sem corte (Figura 3). A elevação no número de panículas por metro quadrado em função do uso de sementes com maior qualidade fisiológica provavelmente foi determinada pelo aumento no número de colmos por metro quadrado (Figura 1 e 2). Já a redução no número de sementes por panícula pode ser atribuída ao aumento no número de panículas por metro quadrado. De acordo com SANGOl et al. (1997), ocorre uma compensação nos componentes de rendimento, quando o acréscimo de um componente é acompanhado por uma redução em outro componente.
$\mathrm{Na}$ maturação, as plantas que foram submetidas ao corte apresentaram menor produção de matéria seca, altura da planta e rendimento de sementes, enquanto o número de colmos e panículas por metro quadrado não diferiram entre os dois tratamentos (Tabela 1). FONTANELI et al. (1994) também constataram a diminuição significativa no rendimento de grãos de aveia-preta nos sistemas em que as plantas foram submetidas ao corte.

\section{CONCLUSÕES}

1) $O$ aumento da qualidade fisiológica de sementes de aveia-preta afeta positivamente 0 número de colmos, a produção de matéria seca, índice de área foliar e o rendimento de sementes.

2) As diferenças na qualidade fisiológica de sementes não causam efeito sobre a capacidade de rebrota. 
SCHUCH, L.O.B. et al. Relação entre a qualidade de sementes...

TABELA 1 - Número de colmos por $\mathrm{m}^{2}$, produção de matéria seca (MS) e altura na maturação, rendimento de sementes, e número de panículas por $\mathrm{m}^{2}$ de plantas de aveia-preta submetidas ao corte.

\begin{tabular}{cccccc}
\hline Corte & Colmos $\mathrm{m}^{-2}$ & $\begin{array}{c}\text { Produção } \\
\text { de MS }\left(\mathrm{kg} \mathrm{ha}^{-1}\right)\end{array}$ & Altura $(\mathrm{cm})$ & $\begin{array}{c}\text { Rendimento de } \\
\text { sementes }\left(\mathrm{kg} \mathrm{ha}^{-1}\right)\end{array}$ & Panículas m $^{-2}$ \\
\hline Sem & $362 \mathrm{a}$ & $3987 \mathrm{a}$ & $118 \mathrm{a}$ & $1512 \mathrm{a}$ & $338 \mathrm{a}$ \\
Com & $340 \mathrm{a}$ & $2999 \mathrm{~b}$ & $90 \mathrm{~b}$ & $1265 \mathrm{~b}$ & $359 \mathrm{a}$
\end{tabular}

${ }^{*}$ Médias seguidas por letras distintas na coluna diferem entre si pelo teste de Duncan a 5\%.

\section{REFERÊNCIAS}

1. BRASIL. Ministério da Agricultura e Reforma Agrária. Secretaria Nacional de Defesa Agropecuária. Regras para análise de sementes. Brasília, 1992. $365 \mathrm{p}$.

2. BRATTACHARYYA, S.; HAZRA, A.K.; SEM-MANDI, S. Accelerated ageing of seeds in hot water: germination characteristics of aged wheat seeds. Seed Science and Technology, v. 13, n. 3, p. 683-690, 1985.

3. BURRIS, J.S. Seed/seedling vigor and field performance. Journal of Seed Technology, v. 1, p. 58-74, 1976.

4. DAN, E.L.; MELLO, V.D.C.; WETZEL, C.T.; POPINIGIS, F.; SOUZA, E.P. Transferência de matéria seca como método de avaliação do vigor de sementes de soja. Revista Brasileira de Sementes, v. 9, n. 3, p. 45-55, 1987.

5. DENARDIN, J.E.; KOCHHANN, R.A. Requisitos para implantação e manutenção do sistema plantio direto. In: CNPTEMBRAPA, FUNDACEP-FECOTRIGO, FUNDAÇÃO ABC. Plantio direto no Brasil. Passo Fundo: Aldeia Norte, 1993. p. 19-23.

6. FLOSS, E.L.O papel da aveia como componente de uma agricultura sustentável. In: REUNIÃO DA COMISSÃO BRASILEIRA DE PESQUISA DE AVEIA, 21., 2001, Lages. Resultados experimentais. Lages: UDESC, 2001. p.1121.

7. FLOSS, E.L.; AUGUSTIN, L.; CALVETE, E. de O.; GRANDO, M.F.; FONTANELI, R.S.; SEVERO, J.L.; RODRIGUES, O. Melhoramento genético de aveia na Universidade de Passo Fundo, 1977/1997. Pesquisa informa, Passo Fundo, ano 1, n. 1, 1999.

8. FONTANELI, R.S.; BASSO, S.M.S; SILVA, G. da; PRATES, L.G.; ROTA, R.; BELAVER, P.R. Avaliação de cereais de inverno para duplo propósito. In: REUNIÃO DA COMISSÃO SULBRASILEIRA DE PESQUISA DE AVEIA, 14., 1994, Porto Alegre. Resultados experimentais. Porto Alegre: Departamento de Plantas de Lavoura/UFRGS, 1994. p. 210215.

9. HÖFS, A. Vigor de sementes de arroz e desempenho da cultura. Pelotas, 2003. 44 f. Tese (Doutorado em Ciência e Tecnologia de Sementes) - Curso de Pós-Gradução em Ciência e Tecnologia de Sementes, Departamento de Fitotecnia, Universidade Federal de Pelotas.

10. KHAN, E.M.; ROBERTS, E.H.; ELLIS, R.H. Effects of seed ageing on growth and yield of spring wheat at different plant-population densities. Field Crops Research, v. 20, p. 175-190, 1989.

11. MACHADO, R.F. Desempenho de aveia branca (Avena sativa L.) em função do vigor de sementes e população de plantas. Pelotas, 2002. 46 f. Dissertação (Mestrado em Ciência e Tecnologia de Sementes) - Curso de Pós-Graduação em Ciência e Tecnologia de Sementes, Departamento de Fitotecnia, Universidade Federal de Pelotas.

12. ROBERTS, E.H. Quantifying seed deterioration. In: MCDONALD Jr., M.B.; NELSON, C.J. (Eds.). Physiology of seed deterioration. Madison:ASA/CSSA/SSSA, 1986. p. 101-123. (Special Publication, 11).

13. SANGOI, L.; ALMEIDA, M.L.; ENDER, M. Vantagens e limitações da utilização de ideotipos no melhoramento de plantas de lavoura. Pesquisa Agropecuária Brasileira, v. 3, n. 1, p. 73-80, 1997.

14. SANTOS, H.S.; REIS, E.M.; POTTKER, D. Culturas de inverno para plantio direto no sul do Brasil. Passo Fundo: EMBRAPA-CNPT, 1990. 24 p. (EMBRAPA-CNPT. Circular Técnica, 3).

15. $\mathrm{SCHUCH,} \mathrm{L.O.B.} \mathrm{Vigor} \mathrm{das} \mathrm{sementes} \mathrm{e} \mathrm{aspectos} \mathrm{da} \mathrm{fisiologia} \mathrm{da} \mathrm{produção} \mathrm{em} \mathrm{aveia-preta} \mathrm{(Avena}$ strigosa Schreb.). Pelotas, 1999. 127 f. Tese (Doutorado em Ciência e Tecnologia de Sementes) - Curso de PósGradução em Ciência e Tecnologia de Sementes, Departamento de Fitotecnia, Universidade Federal de Pelotas.

16. SCHUCH, L.O.B.; LIN, S.S. Atraso na colheita sobre emergência no campo e desempenho de plantas de trigo. Pesquisa Agropecuária Brasileira, v. 17, n. 11, p. 1585-1589, 1982a.

17. SCHUCH, L.O.B.; LIN, S.S. Efeito do envelhecimento rápido sobre o desempenho de sementes e de plantas de trigo. Pesquisa Agropecuária Brasileira, v. 17, n. 8, p. 1163-1170, 1982b.

18. SCHUCH, L.O.B.; NEDEL, J.L; MAIA, M. de S.; ASSIS, F.N. de. Vigor de sementes e adubação nitrogenada em aveiapreta (Avena strigosa Schreb.). Revista Brasileira de Sementes, v. 21, n. 2, p. 127-134, 1999.

19. SCHUCH, L.O.B.; NEDEL, J.L; MAIA, M. de S.; ASSIS, F.N. de. Vigor de sementes e análise de crescimento de aveiapreta. Scientia Agricola, v. 57, n. 2, p. 305-312, 2000.

20. TEKRONY, D.M.; EGLI, D.B.; WICKHAM, D.A. Corn seed vigor effect on no-tillage field performance: II. Plant growth and grain yield. Crop Science, v. 29, p. 1528-1531, 1989.

21. TEKRONY, D.M.; EGLI, D.B. Relationship of seed vigor to crop yield: a review. Crop Science, v. 31, n. 3, p. 816-822, 1991.

Recebido em 24/10/2003

Aceito em 12/11/2007 
